\title{
ENCEINTE DE RADIOPROTECTION EN FER CONSTRUITE AVEC DES RAILS COMME MATERIAU DE BASE
}

\author{
P. BONÉT-MAURY* et M. AUDOUIN** \\ (Manuscrit reçu le 26 novembre 1970 )
}

\begin{abstract}
RÉSUMÉ
Les auteurs proposent l'utilisation de rails usagés pour construire des enceintes de radioprotection en fer, autour des sources importantes de rayonnements ionisants. Ils décrivent la façon dont, avec ces rails, ils ont réalisé, pour un bétatron de $20 \mathrm{MeV}$, une protection biologique qui s'est montré satisfaisante du point de vue technique et économique.
\end{abstract}

\section{ABSTRACT}

The authors suggest to utilize used rails for construction of iron shielded enclosure round large sources of ionizing radiation. They describe how, with this rails, they have realized, for a $20 \mathrm{MeV}$ betatron, a biological shield which appears satisfying from technical and economical point of view.

L'installation d'un bétatron à usage médical comporte la réalisation d'une enceinte de radioprotection, abaissant le débit de dose des photons produits par l'appareil au-dessous des limites maximales admissibles [1].

Cette enceinte est généralement construite en béton et, pour des photons de $20 \mathrm{MeV}$, son épaisseur peut atteindre $200 \mathrm{~cm}$ aux endroits " chauds".

Si le bétatron doit être installé dans les locaux existants d'une clinique ou d'un hôpital, on est souvent obligé, faute de place, de faire appel, pour certaines parties de l'enceinte, à un matériau plus lourd et plus absorbant que le béton (béton lourd, plomb, fer, etc.) pour réduire l'épaisseur du mur de protection. Ces matériaux sont plus coûteux que le béton ordinaire et leur emploi élève très sérieusement le prix de la radioprotection.

L'emploi de rails usagés de chemin de fer permet de réaliser une enceinte d'épaisseur réduite et de prix de revient comparable, ou même dans certains cas, un peu inférieur, à celui d'une construction en béton baryté.

Comme le montre la figure $\mathrm{I}$, les rails sont juxtaposés en succession tête-bêche et imbriqués de façon à laisser entre eux le minimum d'intervalle; ils sont fixés entre eux par soudure électrique.

La figure 2 donne la variation de l'épaisseur de fer le long de l'écran ainsi réalisé; les maximums correspondant à l'axe d'un rail et les minimums aux creux laissés entre deux rails. On supprime pratiquement ces inégalités d'épaisseur en disposant les couches successives de rails de façon à placer les minimums en face des maximums et surtout en remplissant les creux avec un matériau ferreux divisé.

(*) Institut du Radium, in rue Pierre et Marie Curie, Paris Ve. Seine.

(**) Centre de Télécobalthérapie Hartman, I bis, rue des Dames-Augustines, Neuilly-sur-

RADIOPROTECTION, VOL. $6-\mathrm{N}^{\mathrm{O}} 2$ 
Ce matériau est constitué par un mélange de boulons usagés et de petites pastilles d'acier inoxydable, déchets d'usinage. Ce mélange, peu coûteux, a un pouvoir absorbant inférieur à celui du fer, mais supérieur à celui des boulons et des pastilles le composant, comme le montre Ie tableau I donnant la densité et la C.A. I/roe (couche d'absorption $\mathrm{I} / \mathrm{IO}^{\mathrm{e}}$ ) de divers matériaux. Les déterminations C.A. $\mathrm{I} / \mathrm{ro}^{\mathrm{e}}$ ont été faites avec des rayonnements $\gamma$ du cobalt- $60(\mathrm{E}=\mathrm{x}, \mathrm{I} 7$ et $\mathrm{r}, 33 \mathrm{MeV})$ en faisceau large $\left(100 \mathrm{~cm}^{2}\right)$.

\section{TABLEAU I}

\begin{tabular}{|c|c|c|c|c|c|c|c|}
\hline Matériau & $\begin{array}{l}\text { Acier } \\
\text { en } \\
\text { plaque }\end{array}$ & $\begin{array}{c}\text { Rails } \\
+ \\
\text { mélange }\end{array}$ & $\begin{array}{c}\text { Mélange } \\
\text { boulons } \\
+ \text { inox }\end{array}$ & Boulons & $\begin{array}{l}\text { Past. } \\
\text { Inox }\end{array}$ & $\begin{array}{l}\text { Béton } \\
\text { baryté }\end{array}$ & $\begin{array}{l}\text { Béton } \\
\text { ord. }\end{array}$ \\
\hline Densité & 7,2 & 6,4 & 5,3 & 4,8 & 4,5 & $3, \mathrm{I}$ & 2,5 \\
\hline 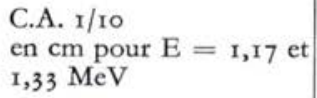 & 5,5 & 7,5 & I0 & 12 & 12,4 & & 17 \\
\hline
\end{tabular}

Pour les photons de $20 \mathrm{MeV}$ les valeurs expérimentales (fer, béton) ou calculées, sont données dans le tableau 2.

TABLEAU 2

\begin{tabular}{l|c|c|c|c}
\hline \hline \multicolumn{1}{c|}{ Matériau } & Acier & $\begin{array}{c}\text { Rails } \\
+ \\
\text { mélange }\end{array}$ & Mélange & Béton \\
\hline & I2 & Is & 20 & 47 \\
$\begin{array}{l}\text { C.A. } 1 / 10^{\mathrm{e}} \\
\text { (en } \mathrm{cm} \text { ) pour } \\
\mathrm{E}=20 \mathrm{MeV}\end{array}$ & & & & \\
\hline \hline
\end{tabular}

Le mélange boulons + inox constitue un matériaux ferreux divisé d'utilisation commode et économique, soit pour remplir les creux d'une protection en fer, comme celle constituée par les rails, soit pour construire des écrans absorbants en le disposant entre deux parois de béton ou d'un autre matériau courant.

Nous avons utilisé les rails et le mélange ferreux divisé pour construire l'enceinte de radiotection d'un bétatron Siemens de $20 \mathrm{MeV}$, installé dans les locaux d'une clinique, dont les dimensions ne permettaient pas de réaliser une protection en béton ordinaire ${ }^{(1)}$. La mise en place des rails et le remplissage des creux avec le mélange a posé quelques problèmes au départ, mais ils ont été résolus et le montage s'est effectué, une fois la technique mise au point, sans difficultés majeures. Les rails constituent une charpente particulièrement robuste, supportant les charges importantes de la protection du plafond. Les figures 3 et 4 montrent les différents stades de la construction.

(1) Le plan de cette enceinte a été établi en collaboration étroite avec l'équipe de radioprotection de Siemens-France; leur contribution a été déterminante pour la réussite de notre projet et nous les en remercions bien vivement. 
Les avantages pratiques et économiques de ce système sont :

I) Gain de temps: la mise en place et la fixation des rails ont été assez rapides : Is rails à l'heure une fois le chantier approvisionné, la difficulté majeure étant la prise du rail par la grue, une grue aimant n'ayant pu être installée. Il n'y a pas de coffrage, évitant les étais énormes et encombrants.

2) Gain de place : les murs ayant une largeur 2,5 fois moindre que ceux avec béton ordinaire, deux fois moindre que ceux avec béton baryté.

3) Solidité : le Bunker construit peut servir de base et de fondation à n'importe quel poids, autant et même plus que la même protection en béton armé, tandis que le béton baryté, mauvais porteur, aurait demandé une armature supplémentaire de béton armé.

4) Economie : plus coûteux que le béton ordinaire, le système est d'un prix de revient équivalent à celui du béton baryté, mais d'un prix inférieur s'il y a des superstructures. De plus, le gain de temps permet de sérieuses économies pour le chantier. En cas de démolition ultérieure, les rails peuvent être dessoudés et vendus à la ferraille au prix d'achat.

5) Esthétique : une fois peintes, les parois apparentes sont d'un effet décoratif moderne très plaisant.

6) Sécurité : on est certain, au départ, de la précision de la protection obtenue, car il n'y a ni poches, ni couches de densités différentes par mélange de béton.

7) Inertie calorifique et insonorisation : elles sont nettement meilleures que celles du béton.

Les contrôles de radioprotection - le bétatron fonctionnant en photons de $20 \mathrm{MeV}$ avec le débit dose maximal de $130 \mathrm{rad} / \mathrm{mn}$ à $6 \mathrm{~s} \mathrm{~cm}$ - ont montré qu'au point le plus " chaud " correspondant à l'impact du faisceau horizontal sur le mur d'enceinte, le débit de dose en photons, à l'extérieur du mur, était de l'ordre de $2 \mathrm{mrem} / \mathrm{h}$ et tombait à quelques $\mathrm{cm}$ de ce point, à 0,2 $\mathrm{mrem} / \mathrm{h}$, la norme étant de $2,5 \mathrm{mrem} / \mathrm{h}$.

Ces matériaux et ces procédés [2] peuvent naturellement être utilisés pour la radioprotection de toute source de rayonnements ionisants : accélérateurs, réacteurs ou radioéléments. Compte tenu des problèmes particuliers d'installation d'une source, ils apparaissent dans de nombreux cas capables de réaliser une enceinte radioprotectrice de façon plus économique et techniquement plus satisfaisante que les matériaux usuels : béton, plomb, terre, eau, etc.

\section{BIBLIOGRAPHIE}

[1] Protection des Travailleurs contre les rayonnements ionisants. Décret no 67.228 du i 5-3-67, J.O. du 22-3-67 et arrêtés du 18-24-4-1968 (J.O. du 8-6-68).

[2] Brevet français $n^{\circ} 6.942 .437$ du 10-12-69. 


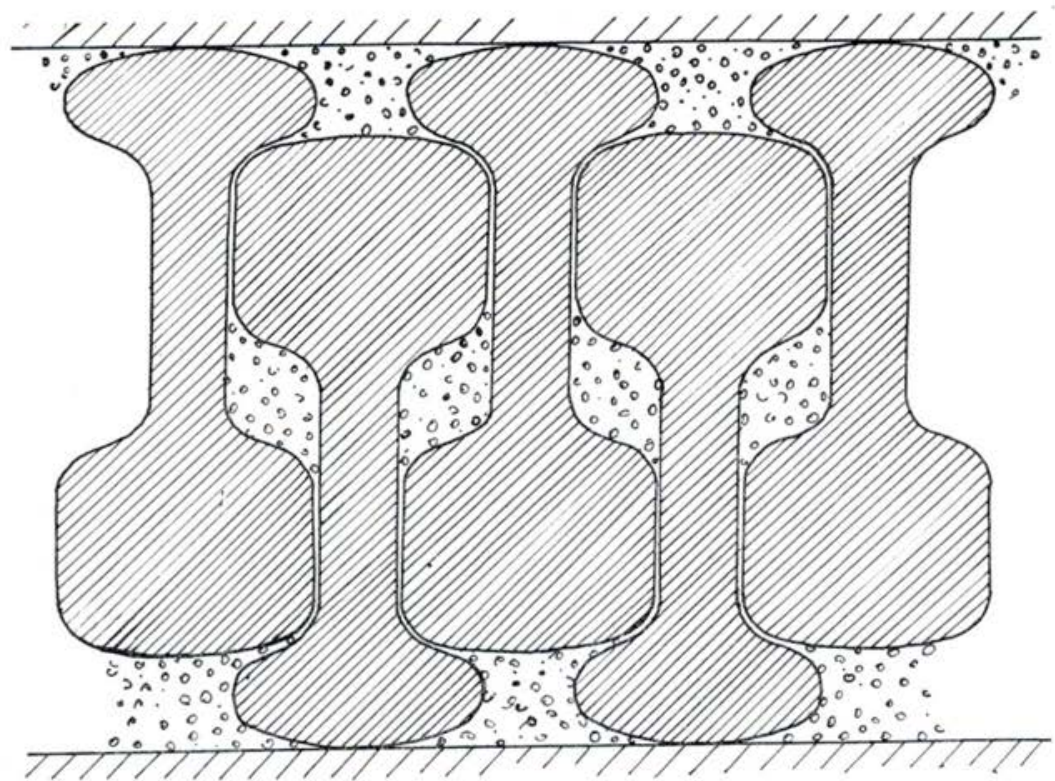

FIG. I. - Juxtaposition des rails en succession tête-bêche.

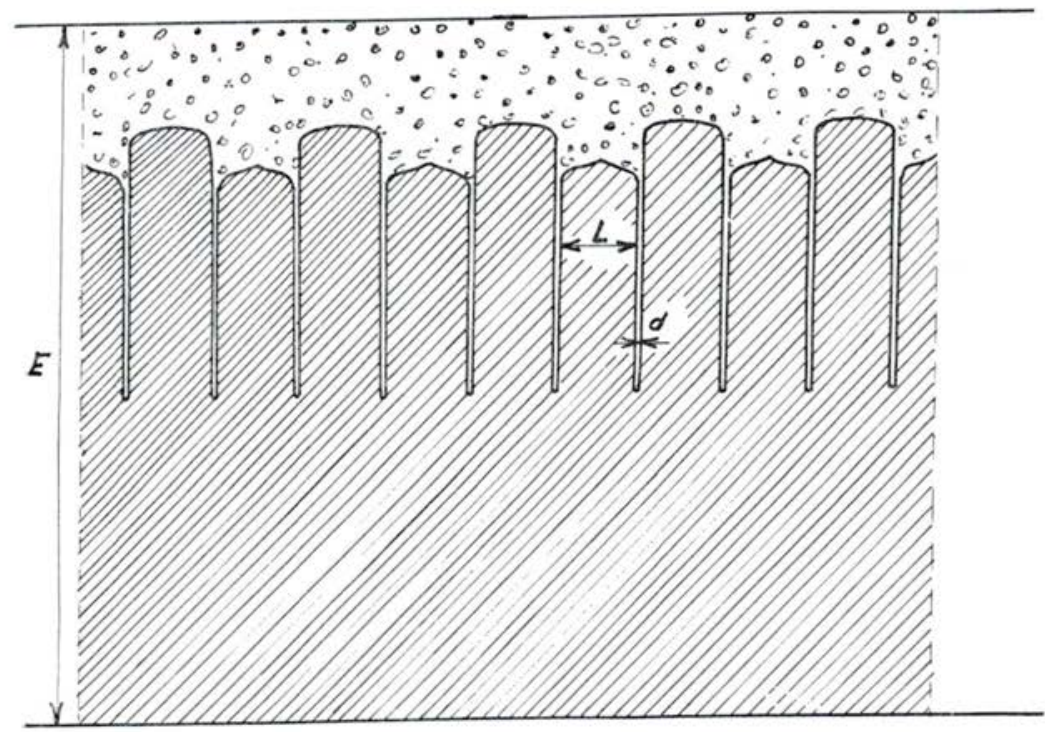

FIG. 2. - Variation de l'épaisseur de fer le long de l'écran réalisé. 


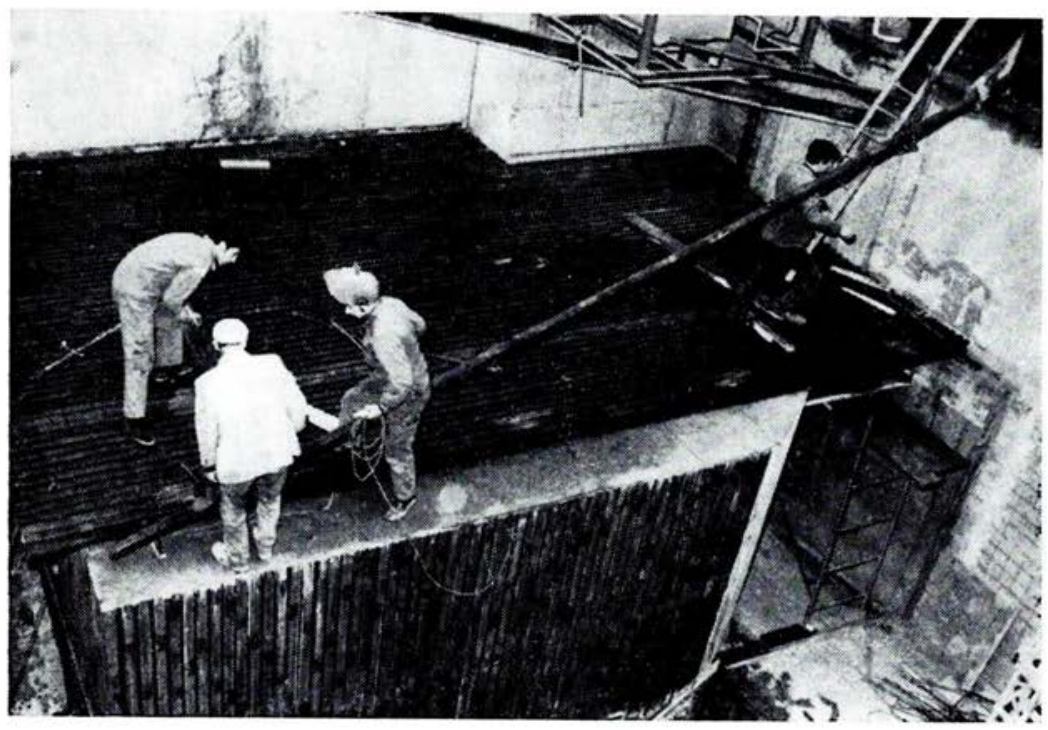

FIG. 3.

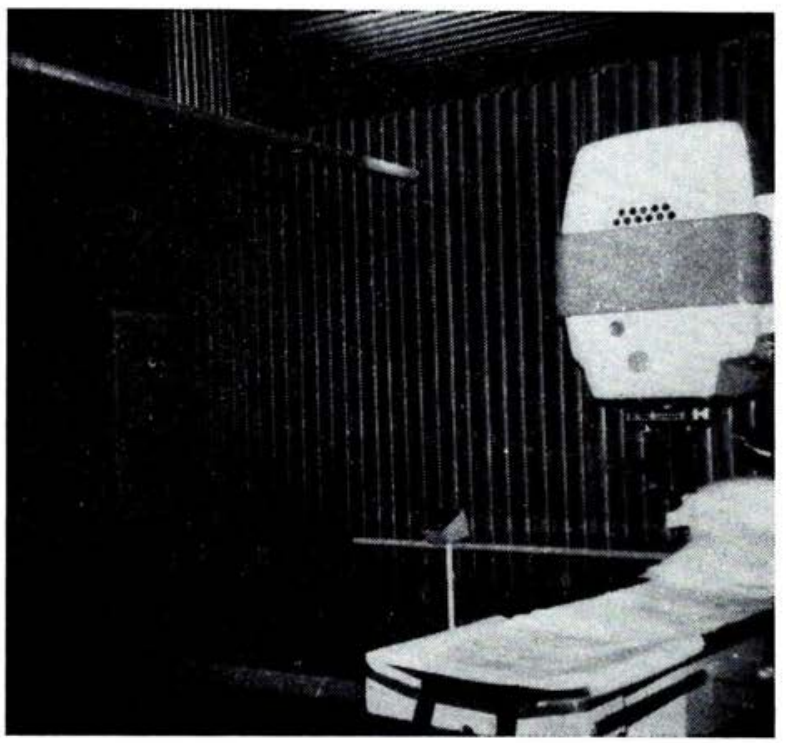

FIG. 4 .

Fig. 3 et Fig. 4. - Construction de l'ouvrage.

VOL. $6-\mathrm{N}^{\circ} 2$ 